\title{
Salud mental relacionada con el trabajo: desafíos para las políticas públicas*
}

Work-Related Mental Health: The Challenges for Public Policies

Recibido: 30 de octubre de 2014 | Aceptado: 15 de junio de 2015

\author{
Marcia Hespanhol Bernardo ** \\ Heloisa APARECIDA DE SOUZA *** \\ JoHANNA GARRIDO PINZÓN ** \\ EDUARDO AlESSANDRO KAWAMURA ${ }^{* * * * *}$ \\ Pontificia Universidad Católica de Campinas, Brasil
}

doi :10.11144/Javeriana.upsy14-5.smrt

Para citar este artículo: Bernardo, M. H., Souza, H. A., Garrido-Pinzón, J., \& Kawamura, E. A. (2015). Salud mental relacionada con el trabajo: desafíos para las políticas públicas. Universitas Psychologica, 14(5), 1613-1624. http://dx.doi.org/10.11144/Javeriana.upsy14-5.smrt

Artículo de reflexión. El desarrollo del artículo contó con el apoyo financiero de CAPES (Coordenação de Aperfeiçoamento de Pessoal de Nível Superior).

*** Profesora investigadora del Programa de Posgrado en Psicología. Correo Electrónico: marciahb@ terra.com.br Teléfono: (55) (19) 33436891

**** Doctoranda del Programa de Posgrado en Psicología.Correo Electrónico: heloisa_apsouza@ yahoo.com.br

****** Doctoranda del Programa de Posgrado en Psicología. Correo Electrónico: johannagarrido28@gmail.com

****** Doctorando del Programa de Posgrado en Psicología. Correo Electrónico: ekawamura@ gmail.com

\section{RES UMEN}

A pesar de que las estadísticas de diversos países indican un aumento significativo de los casos de trastornos mentales relativos al trabajo, las intervenciones propuestas por gobiernos y organizaciones internacionales comúnmente se limitan a los "factores de riesgo psicosocial", con foco en la prevención del estrés. Así las cosas, las acciones son poco críticas frente a los escenarios económicos, políticos y sociales. Este artículo presenta una propuesta más amplia para afrontar los problemas de salud mental ocasionados o agravados por la actividad mental desde el abordaje teórico-metodológico de la Salud Mental relacionada con el Trabajo. Se exponen, asimismo, los principios del campo de la Salud del Trabajador, que hace parte de la política de salud pública brasilera y que orienta actualmente la realización de intervenciones. Palabras Clave

Salud Mental Relacionada con el trabajo; política pública; salud del trabajador, psicología social del trabajo; riesgos psicosociales

\section{A B S T R A C T}

Although statistics from various countries indicate a significant increase in cases of work-related mental disorders, the interventions that governments and international organizations propose are often limited to "psychosocial risk factors" and focused on stress prevention. Also, these interventions are uncritically conducted in relation to contemporary economic, political and social scenarios involving work relations. This article presents a more comprehensive proposal to deal with mental health problems caused and/or aggravated by work activity: the theoretical and methodological approach of 'Work-related Mental Health'. Furthermore, the principles of 'Workers' Health field', part of the Brazilian public health policy, are presented as guidance for conducting these interventions.

Keywords

work-related mental health; public policies; workers' health; work social psychology; psychosocial risks 


\section{Work-Related Mental Health: The Challenges for Public Policies}

A principios de este siglo la Organización Mundial de la Salud (OMS) (2000) ya estimaba que cerca del $40 \%$ de los trabajadores del mundo sufren de algún tipo de trastorno mental. (Organización Mundial de la Salud, 2000). Los datos oficiales de Brasil (Ministério da Previdência e Assistência Social, 2012) y de Colombia (Ministerio del Trabajo, 2013) revelan que los trastornos mentales ocupan el tercer lugar entre las enfermedades laborales que con mayor frecuencia presentan los trabajadores, mientras que las cifras estadísticas de sus órganos oficiales señalan que la incidencia de ese tipo de perjuicios a la salud no para de crecer.

Muchos de estos trastornos están relacionados con el trabajo, como lo confirman diversos autores (Appay, 2005; Dejours, 2000; Franco, Druck, \& Seligamann-Silva, 2010; Venco \& Barreto, 2010). Los aspectos que estos autores apuntan como los más lesivos para la salud mental de los trabajadores son los relativos a las características actuales del trabajo en el mundo globalizado: la precarización creciente y los modelos de organización basados en el toyotismo que, a su vez, poseen otras características como la flexibilización y la individualización de las relaciones de trabajo, la imposición de metas a alcanzar — cada vez más altas-y el envolvimiento de la subjetividad del trabajador en el proceso de trabajo (Antunes, 2000; Bernardo, 2009; Cimbalista, 2007; PulidoMartínez \& Carvajal-Marín, 2013).

Frente a esa realidad, entidades como la Organización Mundial de la Salud (OMS), la Organización Internacional del Trabajo (OIT), la Agencia Europea para la Salud y Seguridad en el Trabajo (EU-OSHA) y otros organismos de los gobiernos de diversos países han manifestado su preocupación e indicado caminos para la prevención y el tratamiento de estos problemas. Para tal fin, diferentes documentos han sido elaborados en las últimas décadas.

A primera vista, esas iniciativas parecen positivas para la sociedad, las empresas y los trabajadores. Sin embargo, a partir del abordaje teóricometodológico crítico adoptado en este artículo
— a saber, la Salud Mental Relacionada con el trabajo-, nos preguntamos si esas acciones buscan abarcar el problema en toda su complejidad o si solo constituyen propuestas paliativas, cuyo objetivo es apenas disminuir la pérdida de productividad en los procesos de trabajo y adaptar a los trabajadores a las condiciones y modelos de organización de trabajo predominantes.

Se entiende que la orientación de las intervenciones que dependen de la iniciativa de los propios empleadores no es suficiente para contrarrestar la enfermedad mental provocada o agravada por el trabajo: se necesitan políticas de Estado que envuelvan la participación directa de los trabajadores. Se parte de la compresión de que la enfermedad y el sufrimiento mental relativos al trabajo precisan ser tratados como una cuestión de salud pública, de tal forma que se estructuren intervenciones que puedan abordar, a profundidad, las causas del problema.

Este artículo tiene como objetivo presentar una perspectiva más amplia, para el desarrollo de posibles políticas públicas orientadas a la promoción de la salud mental de los trabajadores y a la prevención de enfermedades y sufrimiento psíquicos propiciados por la actividad laboral. Para dicho efecto serán presentadas, en primer lugar, propuestas de intervención de algunos países y de la OMS, evidenciando, mediante una postura crítica, las concepciones que sustentan tales propuestas. Posteriormente, se discute el enfoque teórico-metodológico de la Salud Mental Relacionada con el trabajo y se toma en consideración un abordaje que integra diferentes perspectivas y niveles de análisis. El documento cierra con una breve exposición del campo de la Salud del Trabajador desarrollado en Brasil y se presenta como una propuesta de política pública que favorece la realización de intervenciones más efectivas para el problema en cuestión.

Es importante destacar que, a pesar de que el objeto de este artículo no es específicamente la intervención psicológica, la temática aquí abordada alude directamente a la psicología, puesto que distintas propuestas gubernamentales asociadas a ella han sido construidas no solo con la asesoría de psicólogos, sino también con la actuación de estos profesionales en su implementación. 


\section{Algunas perspectivas internacionales sobre la relación entre salud mental y trabajo}

Agencias gubernamentales de varias naciones y reconocidas organizaciones internacionales desarrollaron, en las últimas décadas, estudios que demuestran una preocupación por la salud mental de los trabajadores, señalando el hecho de que las condiciones de trabajo se encuentran directamente relacionadas con el surgimiento de trastornos mentales. No obstante, de un modo en general, se centran en aspectos relacionados con los llamados factores de riesgo psicosocial, cuya identificación se restringe, básicamente, a la utilización de instrumentos cuantitativos con cuestionamientos cerrados. Kristensen (2002), por ejemplo, asocia los factores de riesgo psicosocial a la demanda física, emocional y sensorial del trabajador, a las posibilidades de que el trabajo favorezca el desarrollo y la autonomía del mismo y a las relaciones interpersonales en el lugar de trabajo — que incluyen colegas y jefes-.

Entre las consecuencias para la salud que resultan de los factores de riesgo psicosocial, el más destacado es, sin duda, el estrés. También es tratado de forma bastante genérica, casi como sinónimo de enfermedad o sufrimiento psíquicos. De acuerdo con Pulido-Martínez y Carvajal-Marín (2013), "esta manera de atender a la salud cada día, en mayor medida, coloniza el mundo del trabajo" (p. 73). La Organización Internacional del Trabajo (OIT) asegura que el estrés es la segunda causa más frecuente de trastornos de salud relacionados con el trabajo, siendo responsable de entre el $40 \%$ y $60 \%$ de los días de trabajo perdidos (OIT, 2010).

Tal constatación ha repercutido en las acciones gubernamentales de diversos países, que han buscado ayuda de profesionales especializados — entre ellos psicólogos - para la elaboración de propuestas para afrontar el problema. Dicha preocupación puede ser evidenciada en la realización, por parte órganos de inspección de trabajo de los países integrantes de la Comunidad Europea (European Comission, 2012), de una campaña para la evaluación depara la evaluación de los factores de riesgo psicosocial. Esa acción incluyó entrenamientos, por parte de inspectores de trabajo, para aprender a observar y detectar factores de riesgo psicosocial y la realización de investigaciones por parte de trabajadores, quienes utilizaron instrumentos de evaluación compuestos por cuestionarios.

Con base en algunos datos provenientes de investigaciones sobre el tema, la Agencia Europea para la Salud y Seguridad en el Trabajo (EU-OSHA) elaboró un documento orientado a la gestión del riesgo psicosocial en empresas (European Risk Observatory, 2010). El editorial de dicho documento anuncia que "es necesaria la atención para garantizar que los establecimientos implementen una amplia gama de acciones preventivas en todos los países y sectores", y agrega:

Todo eso sugiere que los factores de "contexto", como estilo gerencial, cultura organizacional y capacidad organizacional, desempeñan un papel importante y ofrecen una ruta potencial para mejorar la gestión de riesgos psicosociales en el local de trabajo en toda Europa. (European Risk Observatory, 2010), p. 9

Esa afirmación permite observar cómo el "contexto" al que refiere la propuesta está restringido al local de trabajo, sin considerar características objetivas de la organización del trabajo —entre ellas el ritmo y metas impuestas — ni la dimensión macrosocial que las determina. Igualmente, es interesante resaltar que uno de los capítulos del referido documento se enfoca en un contexto más general del trabajo en la actualidad. No obstante, ese aspecto no es retomado en las propuestas presentadas. El gobierno de Australia presenta un discurso semejante, por medio de la institución estatal Comcare (2014), responsable por la promoción de la seguridad y la salud de los trabajadores de ese país.

El Centro de Control y Prevención de Enfermedades, órgano de los Estados Unidos de América, también aborda el tema de esa forma. En un documento publicado en Internet (Centers for Disease Control and Prevention, 2011), se discute una serie de aspectos de la salud física y mental de los trabajadores. Sin embargo, el estrés es nuevamente el efecto de mayor incidencia, y las recomendaciones 
descritas para su prevención apenas recaen en el nivel organizacional, sin presentar ningún ejemplo de política nacional.

Entre los países latinoamericanos, Colombia es uno de los que presenta propuestas de evaluación e intervención de riesgo psicosocial para los trabajadores. Estas se encuentran pautadas en la Resolución 2646 de 2008, dictada por el Ministerio de Salud y la Protección Social. Esta resolución tiene por objeto:

(...) establecer disposiciones y definir las responsabilidades de los diferentes actores sociales en cuanto a la identificación, evaluación, prevención, intervención y monitoreo permanente de la exposición a los factores de riesgo psicosocial en el trabajo, así como el estudio y determinación de origen de patologías presuntamente causadas por estrés ocupacional. (2008, p. 1).

En relación con esta norma, consideramos dos aspectos de especial interés. Primero, la norma dice cómo se debe realizar la evaluación, lo cual revela un énfasis en la medición objetiva y subjetiva de los factores de riesgo psicosocial en todos los trabajadores formales, mediante la utilización de instrumentos validados en el país. Segundo, la norma dice quién debe realizar la evaluación: un experto o profesional en psicología - con especialización en Salud Ocupacional, preferiblemente-. Las características de la norma ilustran claramente la sintonía del modelo colombiano con los modelos hegemónicos, propios de los organismos internacionales citados anteriormente, que promueven las buenas prácticas asociadas a la salud mental y el trabajo. Estos modelos aluden fundamentalmente al diagnóstico del estrés, y lo evalúan a través de cuestionarios que se limitan a los ambientes de trabajo y a los aspectos individuales.

En concordancia con esta perspectiva, la OMS (2000) promociona "buenas prácticas" relacionadas con la salud mental y el trabajo. Estas, igualmente, priorizan las iniciativas de los empleadores. El documento versa, inicialmente, sobre la importancia del trabajo para la readaptación e inclusión sociales de las personas con trastornos mentales, reforzan- do así la visión positiva y muchas veces utilitarista asociada al trabajo, sin hacer mínima referencia a la posibilidad de que las condiciones de trabajo desempeñan un papel en el desarrollo de dichos trastornos. Cuando, finalmente, surge la discusión sobre los impactos de las nuevas reglas y estructuras organizacionales, como la globalización, la interdependencia, la automatización y la evolución tecnológica en la salud mental del trabajador, se afirma que, independientemente de los factores causales, el ambiente de trabajo es un espacio propicio para la educación y manutención de la salud mental. Es evidente entonces que la pregunta por la relación entre el problema y el trabajo no se formula en la discusión presentada.

No hay duda de que la inclusión del riesgo psicosocial en las preocupaciones gubernamentales de diferentes países y de importantes agencias nacionales e internacionales, ocupadas del trabajo o de la salud, representa un gran avance. No obstante, la tesis de este artículo es que el diagnóstico del problema tiende a enfocarse esencialmente en las relaciones interpersonales, los factores individuales y en algunos aspectos de la organización del trabajo. Las propuestas de intervención, a su vez, tienden a enfatizar en los cambios que se dan en las relaciones jerárquicas y entre colegas cuando se crean de ambientes de trabajo más amigables y que favorecen la "calidad de vida en el trabajo" (Lacaz, 2009). Sin embargo, no modifican aspectos técnicos que tienen consecuencias directas sobre la salud de los trabajadores, como el ritmo de trabajo, las metas establecidas por la gerencia y el control sobre el trabajo, entre otras (Bernardo, 2009).

Adicionalmente, se observa que los documentos analizados no entienden a los trabajadores como sujetos activos en el proceso de identificación de los factores de riesgo psicosocial o en la proposición de acciones. Por el contrario, son apenas objeto de análisis e intervención. Asimismo, dichos documentos no hacen referencia a las acciones realizadas por órganos públicos, orientadas específicamente a esta temática. En la mayor parte de ellos, las propuestas sirven apenas como indicadores de "buenas prácticas" para los empleadores. 


\section{Las limitaciones de la intervención factores de en riesgos psicosociales}

Es posible afirmar que las propuestas adoptadas para evaluar e intervenir en factores de riesgo psicosocial que presentamos anteriormente parten de los principios simplistas y sesgados de las concepciones tradicionales de la Salud Ocupacional y de la Medicina del Trabajo. Estas, según Nardi (2011), están excesivamente comprometidas con los intereses de las empresas y no con la salud de los trabajadores. La Medicina del Trabajo es una especialidad médica cuyo objetivo es "el bienestar del paciente y la cura de enfermedades a partir del modelo de una práctica liberal, autónoma y dotada de neutralidad afectiva" (Nardi, 2011, p. 328). La Salud Ocupacional, por su parte, "es utilizada predominantemente en las empresas y generalmente es dotada según una lógica positivista orientada a linealidades de tipo causa-efecto, sin considerar la complejidad que la dimensión social y la dimensión subjetiva introducen en las cuestiones de salud" (Seligmann-Silva, 2011, p. 110).

Lacaz (2007) señala que las principales características de esos abordajes se relacionan con la práctica de corte individual y, como "consecuencia de esa comprensión, el control de la salud preconizado por la Salud Ocupacional se resume a la estrategia de adecuar el ambiente de trabajo al hombre y cada hombre a su ambiente de trabajo" (p. 759). Así, el trabajador es apenas objeto de atención a la salud.

Una de las críticas dirigidas a esas perspectivas -que también podría hacérsele a las propuestas de análisis e intervención enfocadas en los factores de riesgo psicosocial—es que están excesivamente comprometidas con los intereses de los empleadores. Y, como resalta Nardi (2011), "la defensa de los intereses del capital puede ocurrir en detrimento de la salud de los trabajadores" (p. 329). En la misma línea está la crítica que Laurell y Noriega (1989) hacen al hecho de que tal perspectiva se base en la categoría de riesgo. De acuerdo con estos autores, tales concepciones definen los riesgos como "agentes nocivos aislados que pueden causar enfermedad (...) registrando, por tanto, elementos aislados entre sí y entre la dinámica global del proceso de trabajo" (pp. 109, 110).

Laurell y Noriega (1989) se inscriben en una corriente llamada Medicina Social Latinoamericana, que consideramos bastante adecuada para sustentar la crítica propuesta en este artículo. Se trata de un abordaje que busca "retornar a lo social para aprehender la determinación de los problemas de la salud de los trabajadores, incorporando categorías del marxismo" (Lacaz, 2007, p. 760). Así, para superar la perspectiva reduccionista enfocada en los riesgos, estos autores defienden que cualquier análisis de la relación entre salud y trabajo debe considerar las condiciones sociohistóricas del capitalismo que determinan las características del trabajo. Para tal fin, proponen la noción de desgaste y afirman que, en el análisis del proceso de trabajo, se deben buscar los "elementos que interactúan dinámicamente entre sí y con el cuerpo del trabajador, generando aquellos procesos de adaptación que se traducen en desgaste, entendido como pérdida de la capacidad potencial y/o efectiva corporal y psíquica" (Laurell $\&$ Noriega, 1989, p. 110). De ese modo, la salud y la enfermedad son vistas como aspectos de un proceso biopsíquico, cuya determinación se asienta en el contexto social. Específicamente, para el caso de los procesos de salud-enfermedad relacionados con el trabajo, Seligmann-Silva (2011) afirma que:

El desgate es visualizado como producto de una correlación desigual de poderes impuestos sobre el trabajo y sobre el trabajador, accionando fuerzas que inciden en el proceso biopsicosocial saludenfermedad. $\mathrm{O}$ mejor, una correlación de poderes y fuerzas en que el ejecutante del trabajo se convierte en perdedor. (p. 135)

Para considerar los complejos elementos del trabajo que provocan el desgaste, Laurell y Noriega (1989) proponen utilizar la categoría de cargas de trabajo. Según estos autores, tales cargas son elementos del proceso de trabajo que, según el contexto sociohistórico, actúan entre sí y con la constitución del cuerpo humano, generando sucesivos y rigurosos procesos de adaptación que pueden ocasionar daños físicos y psíquicos. 
Dicha perspectiva evidencia cuán limitada es la concepción hegemónica de análisis e intervención de los factores de riesgo psicosocial, puesto que hacen énfasis en los aspectos individuales y en las relaciones interpersonales dentro de los ambientes de trabajo sin considerar el contexto más amplio en el cual se sitúan, y tampoco la complejidad de las relaciones allí establecidas.

Ante esta crítica de la concepción hegemónica de la relación entre enfermedad mental y trabajo, presentamos a continuación otra posible forma de intervenir ese desafiante problema. Tal posibilidad está basada en la concepción teórica de la Salud Mental Relacionada con el trabajo (SMRT) propuesta por Seligmann-Silva (2011), considerada más amplia que el abordaje del estrés y más afín a la perspectiva defendida por Laurell y Noriega (1989). Este abordaje comparte los presupuestos del campo de la Salud del Trabajador, que se configura como una política pública integrante del Sistema Único de Salud Brasilero y que nació como una opción para superar la visión restringida y proempleador que había caracterizado, hasta entonces, la Medicina del Trabajo y la Salud Ocupacional.

\section{Salud Mental Relacionada con el trabajo: un abordaje integrador}

La Salud Mental Relacionada con el trabajo (SMRT) propone una comprensión crítica del proceso de sufrimiento y enfermedad mental provocado o agravado por el trabajo y un distanciamiento de las concepciones predominantes en la actualidad. Estas últimas tienden, por una parte, a presentar los modelos de trabajo como naturales y sin posibilidad de cambios y, por otra, a presentar al individuo y su historia familiar como la causa de todos sus problemas psíquicos (Bernardo, Sousa, Garrido-Pinzón, \& Souza, 2015).

De este modo, la perspectiva de la SMRT tiene como principal característica la multidisciplinariedad, puesto que incluye la comprensión de los efectos de la política, de la economía y de las implicaciones socioculturales en la vida de las personas en la contemporaneidad, y adopta, así, una "perspectiva en que las finalidades de las investigacio- nes asumen directrices éticasl" (Seligmann-Silva, 2011, p. 40). Lo anterior presupone una falta de compromiso con los intereses de los empleadores en las evaluaciones y en las intervenciones. Así, los viejos preceptos, encaminados casi exclusivamente a la búsqueda de la productividad, dan lugar a la tentativa de identificar el mayor número de aspectos asociados a la salud-enfermedad que puedan entre los que están la promoción de la salud, pero también intereses políticos, económicos y sociales que potencialmente pueden generar enfermedades asociadas al trabajo-.

Desde esa perspectiva, la salud en general y la salud mental en particular son indisociables. La SMRT propone comprender la salud y la enfermedad no solo como simples estados que se encuentran en posiciones dicotómicas, sino como parte de un proceso complejo en el que ambas coexisten. Así las cosas, la salud "ni es un estado de estabilidad, ni es un estado, ni es estable. La salud es algo que cambia todo el tiempo" (Dejours, 1986, p. 11). Es la armonización de la variabilidad "propia de los procesos psico-orgánicos humanos, inmersos en el transcurso existencial y en la vida social" (Seligmann-Silva, 2011, p. 36).

Partiendo de la concepción de desgaste de Laurell y Noriega (1989), Seligmann-Silva (2011) afirma que, más allá del cuerpo, los "potenciales psíquicos" del trabajador también pueden ser consumidos por el proceso de trabajo y por restricciones vinculadas a él. También explica que "sobre la noción de potenciales psíquicos podemos considerar todos los potenciales que se refieren a la inteligencia y a los sentimientos" (p. 136), configurándose, de esta forma, como desgaste mental. De acuerdo con Paparelli, Sato y Oliveira (2011), "esa definición introduce una aparente simplicidad, que potencializa la interlocución con los diversos profesionales involucrados con SMRT" (p. 125). La idea de desgaste es pensada entonces "como un proceso en el cual están presentes por lo menos dos elementos: el que es desgastado y el que genera desgaste". Sin embargo, y a pesar de su aparente simplicidad, continúan los autores:

1 La cursiva es nuestra 
"el concepto de desgaste guarda la complejidad en la comprensión de los agravantes a la salud mental relacionados al trabajo, una vez que permite superar los límites y los contornos de los diferentes objetos estudiados a partir de distintas lecturas teóricometodológicas" (p. 125).

Discutiendo este enfoque, Maeno y Paparelli (2013) introducen la siguiente cuestión: “¿Cómo identificar los factores laborales de desgaste mental si los trabajadores realizan actividades tan diversas, como, por ejemplo, trabajo en telemarketing, en frigoríficos y en bancos?" (p. 146). Para responderla, las autoras resaltan que "se deben considerar el proceso, las condiciones y la organización del Trabajo" (p. 147), las cuales "tienen como núcleo la cuestión del poder: vivir la salud en el trabajo significa tener la posibilidad de intervenir sobre el propio trabajo" (p. 148).

De este modo, se observa que las cuestiones por las que se inclina la perspectiva de la SMRT no se refieren únicamente a las formas de adaptabilidad de los sujetos, sino también a la necesidad de un cambio radical en las formas básicas de comprensión de los sentidos del trabajo. No se trata, por tanto, de un ejercicio de comprensión del universo individual de los trabajadores, en el que las condiciones y la organización del trabajo se constituyen como un simple telón de fondo, y tampoco de las relaciones interpersonales en los lugares de trabajo. El movimiento es radicalmente distinto: se deben "tomar las relaciones de trabajo y su historicidad como matriz de lectura" (Sato \& Bernardo, 2005, p. 870).

El foco de las investigaciones está puesto sobre la relación entre el trabajo y el proceso saludenfermedad que, más allá de las individualidades, contempla de forma dialéctica los micro y macroescenarios políticos y sociales en los que el trabajo y los trabajadores están inmersos. Seligmann-Silva (2011) propone cinco niveles para analizar las fuentes de determinación de los fenómenos inscritos en la perspectiva interdisciplinaria de la SMRT: el nivel internacional, que incluye las relaciones económicas y políticas en el mundo globalizado; el nivel nacional, que corresponde a las 'políticas industrial, tecnológica, salarial, de salud y educa- cional'; el nivel de las empresas y sus parámetros éticos para las relaciones de trabajo y sus políticas de recursos humanos, entre otras; el nivel microsocial del lugar de trabajo, donde son definidos los aspectos colectivos y las dinámicas intersubjetivas, y finalmente, el nivel individual, “(...) aquel donde el complejo psico-orgánico singular, en su trayectoria

histórica personal, se confronta activamente con fuerzas emanadas de los demás territorios examinados

y penetra en las mallas de sus interacciones" ( $\mathrm{p}$. 118) Vale la pena recordar que, en la perspectiva de los factores de riesgo psicosocial, apenas los dos últimos niveles son tenidos en cuenta.

Se debe destacar que la salud mental representa una dimensión en la cual el cuerpo y las interrelaciones humanas son indisociables; "una precisión aún mayor exige que sea incluido en ese objeto, no solo el trabajo, sino también la falta de trabajo" (Seligmann-Silva, 2011, p. 40) y, con ello, sus efectos devastadores sobre los individuos y sobre la organización colectiva de los trabajadores.

En resumen, la SMRT se fundamenta en una visión distinta del cuerpo para elaborar la relación entre salud mental y trabajo: una que va más allá de la concepción del riesgo psicosocial y del estrés y, por ello, de los parámetros de la Medicina del Trabajo y de la Salud Ocupacional. En concordancia con Paparelli, Sato y Oliveira (2011), decimos que la SMRT permite sustentar, teórica y metodológicamente, acciones en el campo de la Salud del Trabajador, desde el cual se busca superar las perspectivas reduccionistas.

\section{El campo de la Salud del Trabajador}

De acuerdo con Laurell y Noriega (1989), la salud es un proceso que, en el contexto laboral, involucra cargas de trabajo que están directamente asociadas a la relación desigual entre capital y trabajo. Desde esta perspectiva, es claro que no depende únicamente de los empleadores adoptar buenas prácticas para hacer frente a los problemas que ellos mismos están generando. Teniendo en cuenta las particularidades del contexto contemporáneo y el "nuevo espíritu del capitalismo" (Boltanski \& 
Chiapello, 2002) que lo acompaña, hacerlo sería como pedirle a un zorro que cuidase de un gallinero. Esto resulta aún más problemático en países de economía dependiente, donde nunca tuvo lugar un "Estado de bien-estar" (Comín, 2007), como Brasil y Colombia. Así las cosas, son necesarias políticas públicas efectivas para enfrentar los problemas de salud pública y que estas incluyan la participación de los trabajadores en el análisis y en la elaboración de las propuestas de intervención, como bien ha señalado Martínez-Alcántara (2007).

En este sentido, consideramos que el campo teórico-práctico de la Salud del Trabajador (Lacaz, 2007) desarrollado en Brasil es una base propicia para la realización de acciones encaminadas a proteger la salud mental de los trabajadores, aun teniendo en cuenta todas las limitaciones que están siendo experimentadas para su efectiva implementación en el contexto neoliberal actual, conforme señala Campos (2007).

El campo de la Salud del Trabajador tiene como una de sus bases las proposiciones de Laurell y Noriega (1989). Con ello, se busca comprender la relación entre el proceso salud-enfermedad y el trabajo en toda su complejidad. Ese campo, contrario en sus principios a la Medicina del Trabajo y a la Salud Ocupacional, emergió en el contexto del movimiento sindical en Brasil a finales de los setenta. Está constituido por los pilares de la producción académica, por la programación en salud en la red pública y por el movimiento de los trabajadores (Lacaz, 2007; Sato \& Bernardo, 2005).

Tal como afirman Paparelli, Sato y Oliveira (2011), la Salud del Trabajador cuestiona la simplificación ideológica que atribuye a los individuos y a sus características personales las causas de sus problemas de salud. En cambio, tiene en cuenta la amplia complejidad de la historicidad, los aspectos sociales, económicos, organizacionales y los procesos psicosociales inmersos en el proceso salud-enfermedad. Para Lacaz (2007), "el enfoque de la Salud del Trabajador busca rescatar el lado humano del trabajo y su capacidad protectora contra los agravantes a la salud de los trabajadores, tales como malestares, incomodidades, desgastes, yendo más allá de los accidentes y enfermedades". Lacaz hace hincapié, así, en el rescate del "real ethos del trabajo: libertario y emancipador" (p. 760).

Entre los fundamentos teórico-metodológicos que configuran el campo de la Salud del Trabajador está el llamado Movimiento Obrero Italiano (Oddone, Marri, Gloria, Briante, Chiattella, \& Re, 1986), que surgió en el seno del movimiento sindical en la década del sesenta, en Italia, y que contó con la participación de psicólogos. Su influencia más importante en el campo de la Salud del Trabajador, en Brasil, está en "la valorización del saber y de la subjetividad del trabajador en el proceso de identificación de factores perjudiciales para la salud en el lugar de trabajo y en la lucha por cambios" (Bernardo, Souza, Dassan, \& Garbin, 2013, p. 178). Esta propuesta tiene como principio que el saber práctico de los trabajadores es tan importante como el saber técnico de los profesionales y que, por tanto, deben sumarse a la búsqueda de mejores condiciones de salud en el trabajo, en lugar de que el saber técnico niegue al de los trabajadores.

Especialmente y en relación con las intervenciones, las propuestas de la política pública de la Salud del Trabajador — definidas en la definidas en la Constitución Federal Brasilera (Brasil, 1988) y en la Ley 8080 que regula su sistema de salud (Brasil, 1990) — abarcan básicamente tres niveles no aislables entre sí. El primero es la asistencia a la salud de trabajadores que se han enfermado o accidentado en el trabajo. El segundo comprende acciones de prevención, mediante la vigilancia en ambientes de trabajo por parte equipos multidisciplinarios de salud pública y con la participación de trabajadores, para identificar aspectos que puedan perjudicar su salud o poner sus vidas en riesgo. La última modalidad de acción está encaminada a la promoción de salud por medio de la concientización de los trabajadores y de la población general sobre aspectos del trabajo que puedan ocasionar o agravar problemas de salud (Ley 8080, 1990; Lacaz, 2005; Campos, 2007; Bernardo \& Garbin, 2011).

De acuerdo con esta propuesta, cualquier evaluación e intervención - ya sea individual o colectiva - asociada a la Salud Mental Relacionada con el trabajo debe superar las acciones que limitan los factores de riesgo psicosocial al estrés. El trabajador debe ser sujeto activo en la identificación 
de las fuentes generadoras de enfermedad y en la elaboración de propuestas para superarlas. Para tal efecto, la organización colectiva de los trabajadores —que se ha dado históricamente por medio de sindicatos - es fundamental. Por eso, entre las actividades de la Salud del Trabajador debe estar la promoción de un debate crítico — del que participen los trabajadores- sobre sus condiciones de trabajo e incentivar acciones colectivas para lograr cambios en niveles más amplios. Tal propuesta coincide con la de Martínez-Alcántara y HernándezSánchez (2005):

(...) es fundamental dotar a los trabajadores de un mayor control sobre su proceso laboral. Es decir, restituirles la capacidad para decidir el ritmo al cual se debe desarrollar el trabajo, el momento en que debe ser suspendida la actividad, etcétera, así como planear espacios de participación de los trabajadores para la toma de decisiones del propio trabajo desarrollado e involucrarlos en la generación de propuestas novedosas de reorganización del trabajo. Sin lugar a dudas estas acciones permitirán recuperar parte del conocimiento que los propios trabajadores tienen sobre las actividades que cotidianamente realizan y se abrirá un espacio para que puedan asumirse como sujetos activos de un trabajo que seguramente tendrán que desarrollar durante toda su vida. (p. 343)

Es evidente que el tipo de intervención que propone la Salud del Trabajador tiene como horizonte un cambio en las relaciones de poder que existen en el mundo del trabajo. De ello se desprende una comprensión de la salud como acción política y un cambio radical del lugar del psicólogo, quien, en vez de intervenir sobre los individuos, contribuye a la concientización sobre las relaciones que permean el mundo del trabajo (Martín-Baró, 1989).

La efectividad de las intervenciones sobre la SMRT, desde esta perspectiva, es un gran desafío en la actualidad, teniendo en cuenta la predominancia del neoliberalismo en el mundo del trabajo. Aun así, creemos que debemos aspirar a políticas públicas que integren los principios que orientan el campo de la Salud del Trabajador, realizando acciones que vayan más allá de prácticas paliativas e inocuas, que sirven apenas para garantizar la productividad y no el bienestar y la salud de los trabajadores.

\section{Consideraciones Finales}

Ante toda la complejidad de los problemas de la Salud Mental Relacionada con el trabajo, concluimos que no existen soluciones fáciles. Este artículo buscó evidenciar la gravedad del asunto y presentar, a su vez, una perspectiva más compleja de intervención que pueda promover políticas públicas orientadas a la salud mental de los trabajadores.

De acuerdo con Brigagão, Nascimento y Spink (2011), "hablar de política pública remite a reconsiderar cuestiones sobre el contexto de construcción de esas políticas y acerca de los actores que están involucrados en esa red heterogénea” (p. 200). Así, con base en la perspectiva teórica de la Salud Mental Relacionada con el trabajo y del campo de la Salud del Trabajador, consideramos necesaria una fuerte conexión entre saberes y quehaceres multidisciplinarios, no solamente en la aplicación de políticas, sino en la participación de su elaboración. Investigadores y profesionales de las diversas áreas asociadas a la Salud Mental y el Trabajo deben comprender los contextos sociales, culturales y económicos de los individuos, valorar el saber de los trabajadores y actuar con compromiso político y principios éticos, yendo más allá de la aplicación de las técnicas y saberes de su especialización, subordinadas a los dictámenes del poder económico.

Entre los profesionales convocados a cambiar radicalmente su postura frente al status quo están los psicólogos, quienes deben examinar cuidadosamente las implicaciones que produce su conocimiento y práctica psicológicas (Pulido-Martínez \& CarvajalMarín, 2013). Esto aún más en los escenarios en que esta disciplina ha sido invitada a legitimar el campo de los riesgos psicosociales en el trabajo.

De esta forma $-\mathrm{y}$ sin olvidar que la Salud Mental Relacionada con el trabajo pertenece a un contexto social más amplio, se hace necesario reemplazar acciones puntuales de 'intervención' psicológica en el campo del trabajo — las cuales, desde abordajes reduccionistas, convierten los problemas políticos y de poder en problemas psicológicos (Pri- 
lleltensky, 1994) — por prácticas integradoras, que aboguen por un diálogo entre los aspectos micro y macro que circundan el mundo del trabajo.

\section{Referencias}

Antunes, R. (2000). Os sentidos do trabalho. São Paulo: Boitempo.

Appay, B. (2005). La dictadure du succés. Paris: L'Hammartan.

Bernardo, M. H. (2009). Discurso flexível, trabalho duro: o contraste entre a vivência de trabalhadores e o discurso de gestão empresarial. São Paulo: Expressão Popular.

Bernardo, M. H., \& Garbin, A. C. (2011). A atenção à saúde mental relacionada ao trabalho no SUS: desafios e possibilidades. Revista Brasileira de Saúde Ocupacional, 36(123), 103-117.

Bernardo, M. H., Sousa, C. C., Garrido-Pinzón, J., \& Souza, H. A. (2015). A práxis da Psicologia Social do Trabalho: reflexões sobre possibilidades de intervenção. En M. Chalfin, O. Furtado, \& T. R. Raitz (Orgs.), Psicologia Social e Trabalho: perspectivas críticas (pp. 16-39). Florianópolis: Abrapso Editora/Edições do Bosque.

Bernardo, M. H., Souza, H. A., Silva, D. L. D., \& Garbin, L. S. (2013). A Psicologia e a Saúde do Trabalhador: Para além da prática hegemônica. En M. H. Bernardo, R. L. Guzzo \& V. S. Trevisan (Orgs.), Teorias e práticas psicossociais nos contextos de comunidades, trabalho e educação (pp. 171-189). Campinas: Alínea.

Brasil (1988). Constituição da Republica Federativa do Brasil. Publicada no Diário Oficial da União em 5 de outubro de 1988.

Brasil (1990). Lei no 8080 (Lei Orgânica da Saúde). Brasília, DF: Ministério da Saúde.

Brigagão, J., Nascimento, V. L. V., \& Spink, P. (2011). As interfaces entre Psicologia e políticas públicas e a configuração de novos espaços de atuação. Revista de Estudos Universitários, 37(1), 199-215.

Boltanski, L., \& Chiapello, E. (2002). El nuevo espíritu del capitalismo. Madrid: Akal.

Campos, G. W. S. (2007). O SUS entre a tradição dos Sistemas Nacionais e o modo liberal-privado para organizar o cuidado à saúde. Ciência E\& Saúde Coletiva, 12, 1865-1874.
Centers for Disease Control and Prevention (2011). Mental Health and Chronic Disease in the Workplace. Recuperado de http://www.cdc.gov/nationalhealthyworksite/docs/nhwp_mental_health_ and_chronic_disease_combined_3.pdf.

Cimbalista, S. (2007). Sufrimiento: los significados adversos de las condiciones de trabajo en el sistema de producción flexible. Revista Universitas Psychologica, 6(1), 69-78.

Comín, F. (2007). El surgimiento y desarrollo del Estado del Bienestar (1883-1980). En S. Salort i Vives \& R. H. Muñoz (Eds.), El estado del bienestar en la encrucijada (pp. 67-128). Madrid: Publicaciones de la Universidad de Alicante.

Comcare (2014). Work-related MentalStress. Australian Government. Recuperado de https://www.comcare.gov.au/preventing/hazards/psychosocial_hazards/work-related_mental_stress

Dejours, C. (1986). Por um novo conceito de saúde. Revista Brasileira de Saúde Ocupacional, 54(14), 7-11.

Dejours, C. (2000). A banalização da injustiça social. Rio de Janeiro: Editora FGV.

European Risk Observatory (2010). Management of psychosocial risks at work: An analysis of the findings of the European survey of enterprises on New and emerging risks. European Agency for Safety and Health at Work (EU-OSHA).

Franco, T., Druck, G., \& Seligmann-Silva, E. (2010). As novas relações de trabalho, o desgaste mental do trabalhador e os transtornos mentais no trabalho precarizado. Revista de Saúde Ocupacional, 35(122), 229-248.

Kristensen, T. S. (2002). A new tool for assessing psychosocial factors at work: The Copenhagen Psychosocial Questionnaire. TUTB Newsletter September, 19-20, 45-47.

Lacaz, F. A. C. (2005). Saúde do Trabalhador: novasvelhas questões. Ciência E⿱ Saúde Coletiva, 10(4), 797-807.

Lacaz, F. A. C. (2007). O campo Saúde do Trabalhador: resgatando conhecimentos e práticas sobre as relações trabalho-saúde. Cadernos de Saúde Pública, 23(4), 757-766.

Lacaz, F. A. C. (2009). Qualidade de vida no trabalho: um conceito político e polissêmico. Trabalho, Educação e Saúde, 7, 565-572. 
Laurell, A. C., \& Noriega, M. (1989). Processo de Produção e saúde: trabalho e desgaste operário. São Paulo: Hucitec.

Ley n 8080 (1990). Lei Orgânica da Saúde. Dispõe sobre as condições para a promoção, proteção e recuperação da saúde, a organização e o funcionamento dos serviços correspondentes e dá outras providências. Ministério da Saúde, 1990. Brasil.

Maeno, M., \& Pararelli, R. (2013). O trabalho como ele é e a saúde mental do trabalhador. En M. A. Silveira (Org.), Inovação para o desenvolvimento de organizações sustentáveis: trabalho, fatores psicossociais e ambiente saudável (pp. 145-166). Campinas, Centro de Tecnologia da Informação Renato Archer (CTI).

Martín-Baró, I. (1989). Psicología política del trabajo en América Latina. Revista de Psicología de El Salvador, 8(31), 5-25.

Martínez-Alcántara, S. (2007). La investigación participativa como práctica social y su aportación al mundo laboral a través del modelo obrero. Revista Salud de los Trabajadores, 15(2), 107-117.

Martínez-Alcántara, S., \& Hernández-Sánchez, A. (2005). Necesidad de estudios y legislación sobre factores psicosociales en el trabajo. Revista Cubana de Salud Pública, 31(4), 338-44.

Ministério da Previdência e Assistência Social (2012). Cai número de acidentes de trabalho e aumenta afastamentos por transtornos mentais. Informativo Eletrônico do Ministério da Previdência Social, 59. Recuperado de http://www.previdencia.gov.br/arquivos/office/4_120326-105114-231.pdf.

Resolución no 002646 (2008). Establece disposiciones y se definen responsabilidades para la identificación, evaluación, prevención, intervención y monitoreo permanente de la exposición a factores de riesgo psicosocial en el trabajo y para la determinación del origen de las patologías causadas por el estrés ocupacional. Ministerio de la Protección Social. Diario Oficial 47059 de julio 23 de 2008. Recuperado de http://www.alcaldiabogota.gov.co/sisjur/ normas/Norma1.jsp?i=31607.

Ministerio del Trabajo. (2013). Informe Ejecutivo de la Segunda Encuesta Nacional de Condiciones de Se- guridad y Salud en el Trabajo en el Sistema General de Riesgos Laborales de Colombia. Recuperado de: http://ccs.org.co/salaprensa/images/Documentos/ INFORME_EJECUTIVO_II\%20ENCSS.pdf.

Nardi, H. C. (2011). Saúde do Trabalhador. En A. D. Cattani \& L. Holzmann (Orgs.), Dicionário crítico de trabalho e tecnologia (pp. 326-330). Porto Alegre: Zouk.

Oddone, I., Marri. G., Gloria, S., Briante, G., Chiattella, M., \& Re, A. (1986). Ambiente de trabalho: a luta dos trabalhadores pela saúde. São Paulo: Hucitec.

Organização Internacional do Trabalho. (2010). Riesgos emergentes y nuevos modelos de prevención en un mundo de trabajo en transformación. Ginebra: OIT.

Organización Mundial de la Salud (2000). Mental health and work: impact, issues and good practices. Geneva: World Health Organization. Recuperado de http:// www.who.int/whr/2001/en/whr01_po.pdf.

Pulido-Martínez, H. C., \& Carvajal-Marín, L. M. (2013). Riesgos psico-laborales y subjetividad: tensiones contemporáneas entre patologización y despatologización en los discursos psicoló-gicos. Revista Quaderns de Psicologia, 15(1), 69-80.

Paparelli, R., Sato, L., \& Oliveira, F. (2011). A Saúde Mental relacionada ao trabalho e os desafios aos profissionais da saúde. Revista Brasileira de Saúde Ocupacional, 36(123), 118-127.

Prilleltensky, I. (1994). The morals and politics of psychology: Psychological discourse and the status quo. New York: State University Press.

Sato, L., \& Bernardo, M. H. (2005). Saúde mental e trabalho: os problemas que persistem. Ciência Saúde Coletiva, 10(4), 869-878.

Seligmann-Silva, E. (2011). Trabalho e desgaste mental: o direito de ser dono de si mesmo. São Paulo: Cortes.

European Comission (2012). Committee of Senior Labour Inspectors Annual Report. Recuperado de http://ec.europa.eu/transparency/regexpert/index.cfm?do=groupDetail. groupDetailDoc\&id=10037\&no=1

Venco, S., \& Barreto, M. (2010). O sentido social do suicídio no trabalho. Revista Espaço Acadêmico, 9(108), 1-8. 
